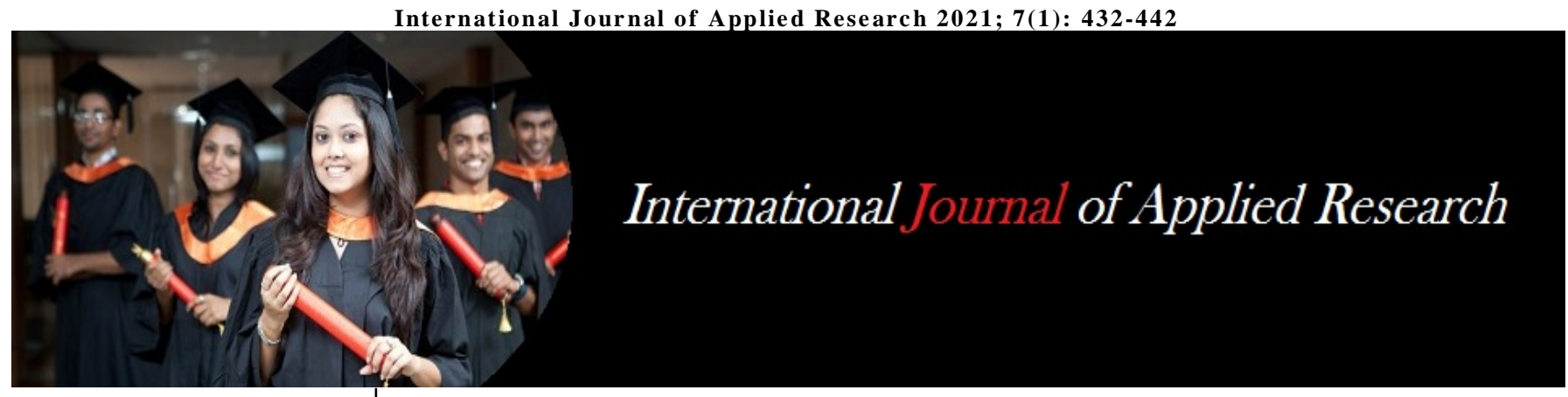

ISSN Print: 2394-7500

ISSN Online: 2394-5869

Impact Factor: 8.4

IJAR 2021; 7(1): 432-442

www.allresearchjournal.com

Received: 25-11-2020

Accepted: 29-12-2020

Arun Kumar Chaudhary

Department of Management

Science (Statistics), Nepal

Commerce Campus, Tribhuvan

University, Kathmandu, Nepal

Vijay Kumar

Department of Mathematics

and Statistics, DDU

Gorakhpur University,

Gorakhpur, Uttar Pradesh,

India
Corresponding Author: Arun Kumar Chaudhary Department of Management Science (Statistics), Nepal

Commerce Campus, Tribhuvan University, Kathmandu, Nepal

\section{Arctan exponential extension distribution with properties and applications}

\author{
Arun Kumar Chaudhary and Vijay Kumar
}

DOI: https://doi.org/10.22271/allresearch.2021.v7.i1f.8251

\section{Abstract}

In the presented work, a continuous distribution named arctan exponential extension distribution has been introduced along with relevant properties which are statistically and mathematically derived and analyzed. While estimating the model parameters, three well-known estimation methods are employed which are Cramer-Von-Mises (CVM), maximum likelihood estimation (MLE) and least-square estimation (LSE) methods. With the help of a real set of data, in comparison to existing models, the presented distribution's goodness-of-fit is evaluated.

Keywords: Arctan distribution, exponential extension distribution, survival function, MLE

\section{Introduction}

Lifetime distributions are generally used to study life span of components of a system and in general, reliability and survival analysis. Lifespan distributions are commonly used in areas such as life science, medicine, biology, engineering, insurance, etc. Various continuous probability distributions for instance Weibull, Exponential and Gamma have been commonly applied to examine lifespan data. For a few years, most of the researchers are attracted towards the exponential distribution for its likely in modeling lifespan data, and it has been perceived that this distribution has done outstandingly in several uses due to the existence of closed form solutions to many survival analyses. It can easily be justified under the supposition of constant failure rate but in the practice, the failure rates are not always constant. Therefore, haphazard use of exponential lifetime model seems to be inappropriate and unrealistic. In modern years, novel classes of models have been introduced centered on adjustments of the existing classical probability models, Marshall and Olkin (2007).

Newly, several efforts have been made to generate novel distributions to lengthen recognized distributions as well as to deliver unlimited flexibility for data encountered. Some procedures might be used to form a greater family by including additional parameters to an established distribution. So, some models with addition of extra parameters to produce novel models have been recommended in the statistical literature, Rinne (2009) and Pham and Lai (2007).

For a few decades, it is found that the exponential distribution is taken as base distribution for generating novel family of distribution. The modifications of the exponential distribution were introduced by different researchers, some of them are, beta exponential (Nadarajah and Kotz, 2006) ${ }^{[27]}$, Gupta and Kundu (2007) ${ }^{[12]}$ have presented the generalized exponential

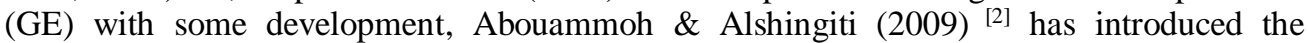
reliability estimation of the generalized inverted exponential distribution, beta GE (BarretoSouza et al., 2010) ${ }^{[4]}$, Exponential Extension (EE) distribution (Kumar, 2010) ${ }^{[18]}$, KW (Kumaraswamy) exponential (Cordeiro and de Castro, 2011) ${ }^{[10]}$, Nadarajah \& Haghighi (2011) ${ }^{[26]}$ have presented an extension of the exponential distribution, gamma EE by (Ristic and Balakrishnan, 2012) ${ }^{[30]}$, Transmuted EE distribution by (Merovci, 2013) ${ }^{[24]}$, Gomez et al. (2014) ${ }^{[14]}$ have presented a new extension of the exponential distribution. Mahdavi \& Kundu (2017) ${ }^{[22]}$ have presented a novel technique in order to generate new distribution via an application to exponential distribution. Recently, the Alpha power transformed extended exponential distribution have introduced by (Hassan et al., 2018) ${ }^{[15]}$. Almarashi et al. (2019) ${ }^{[3]}$ have presented a new extension of exponential distribution with some statistical properties. Abdulkabir \& Ipinyomi, (2020) ${ }^{[1]}$ have introduced the Type II half-logistic exponentiated 
exponential distribution. Joshi and Kumar (2020) ${ }^{[16]}$ have created half-logistic NHE distribution. Joshi \& Kumar (2020) ${ }^{[17]}$ presented the Lindley exponential power. Chaudhary and Kumar (2020) ${ }^{[6]}$ have presented the half logistic exponential extension distribution using the parent distribution as exponential extension distribution. The truncated Cauchy powerexponential distribution was introduced by (Chaudhary et al., 2020) ${ }^{[7]}$ exponential distribution as base distribution. Chaudhary and Kumar (2020) ${ }^{[8]}$ have presented another flexible survival model using exponential extension called logistic exponential extension model. A new exponentiated exponential extension distribution was introduced by Chaudhary and Kumar (2020) ${ }^{[9]}$ whose hazard rate function can have various shapes.

Gómez-Déniz and Calderín-Ojeda (2015) ${ }^{[13]}$ have presented the arctan distribution which was used to model Norwegian fire insurance data. The CDF and PDF of arctan family of distribution with support [a, b] is given by

$$
\begin{aligned}
& F(x)=1-\frac{\arctan [\alpha\{1-G(x)\}]}{\arctan (\alpha)} ; x \geq 0, \alpha>0 ; \alpha \in[\text { a, b] } \\
& f(x)=\frac{1}{\arctan (\alpha)} \frac{\alpha g(x)}{1+[\alpha\{1-G(x)\}]^{2}} ; x \geq 0, \alpha>0
\end{aligned}
$$

\section{Here $G(x)$ and $g(x)$ are the CDF and PDF of any parent distribution}

Here the main aim is to introduce a model with higher flexibility by adding just one extra parameter to the exponential extension model to achieve a good fit to real data. We illustrated the properties of the arctan exponential extension distribution and illustrate its applicability. The article follows the following structure. The new arctan exponential extension distribution is introduced along with discussion of its distributional properties in Section 2. Different estimations methods are introduced and used to derive parameter estimation in section 3. In Section 4 we have considered two real sets of data to analyze and explore the applications of the proposed model. Here we give the ML estimators of the parameters and approximate confidence intervals also for the above-mentioned method of estimations. Assessment of validity of the model is also done. Then, Section 5 gives conclusion.

\section{The Arctan Exponential Extension (ATEE) Distribution}

Nadarajah \& Haghighi (2011) ${ }^{[26]}$ has defined the exponential distribution's extension called as exponential extension distribution. The CDF of this model can be defined as

$\mathrm{G}(x)=1-\exp \left\{1-(1+\lambda x)^{\beta}\right\} ; x \geq 0,(\beta, \lambda)>0$

The corresponding PDF is

$$
g(x)=\alpha \lambda(1+\lambda x)^{\beta-1} \exp \left\{1-(1+\lambda x)^{\beta}\right\} ; x \geq 0,(\beta, \lambda)>0
$$

The CDF and PDF of Arctan generalized exponential distribution with parameters $\alpha, \beta$ and $\lambda$ is acquired using the equations (1.1) and (1.2) where parent distributions are (2.1) and (2.2) and can be expressed as

$$
\begin{aligned}
& F(x)=1-\frac{\arctan \left[\alpha \exp \left\{1-(1+\lambda x)^{\beta}\right\}\right]}{\arctan (\alpha)} ; x \geq 0,(\alpha, \beta, \lambda)>0 \\
& f(x)=\frac{\alpha \beta \lambda}{\arctan (\alpha)} \frac{(1+\lambda x)^{\beta-1} \exp \left\{1-(1+\lambda x)^{\beta}\right\}}{1+\left\{\alpha \exp \left\{1-(1+\lambda x)^{\beta}\right\}^{2}\right.} ; x \geq 0,(\alpha, \beta, \lambda)>0
\end{aligned}
$$

\section{Reliability function}

ATEE distribution's reliability function is

$$
R(x)=1-F(x)=\frac{\arctan \left[\alpha \exp \left\{1-(1+\lambda x)^{\beta}\right\}\right]}{\arctan (\alpha)}
$$

\section{Hazard function}

ATEE distribution's failure rate function is

$$
h(x)=\frac{f(x)}{R(x)}=\frac{\alpha \beta \lambda}{\arctan \left[\alpha \exp \left\{1-(1+\lambda x)^{\beta}\right\}\right]} \frac{(1+\lambda x)^{\beta-1} \exp \left\{1-(1+\lambda x)^{\beta}\right\}}{1+\left\{\alpha \exp \left\{1-(1+\lambda x)^{\beta}\right\}^{2}\right.} ; x \geq 0,(\alpha, \beta, \lambda)>0
$$




\section{Reversed Hazard function is}

$R h(x)=\frac{f(x)}{F(x)}=\frac{\alpha \beta \lambda}{\arctan (\alpha)-\arctan \left[\alpha \exp \left\{1-(1+\lambda x)^{\beta}\right\}\right]} \frac{(1+\lambda x)^{\beta-1} \exp \left\{1-(1+\lambda x)^{\beta}\right\}}{1+\left\{\alpha \exp \left\{1-(1+\lambda x)^{\beta}\right\}^{2}\right.} ; x \geq 0,(\alpha, \beta, \lambda)>0$

In Figure 1, illustration of graphs of hazard rate function and PDF of ATEE distribution for varying $\alpha$, and $\beta$ values.
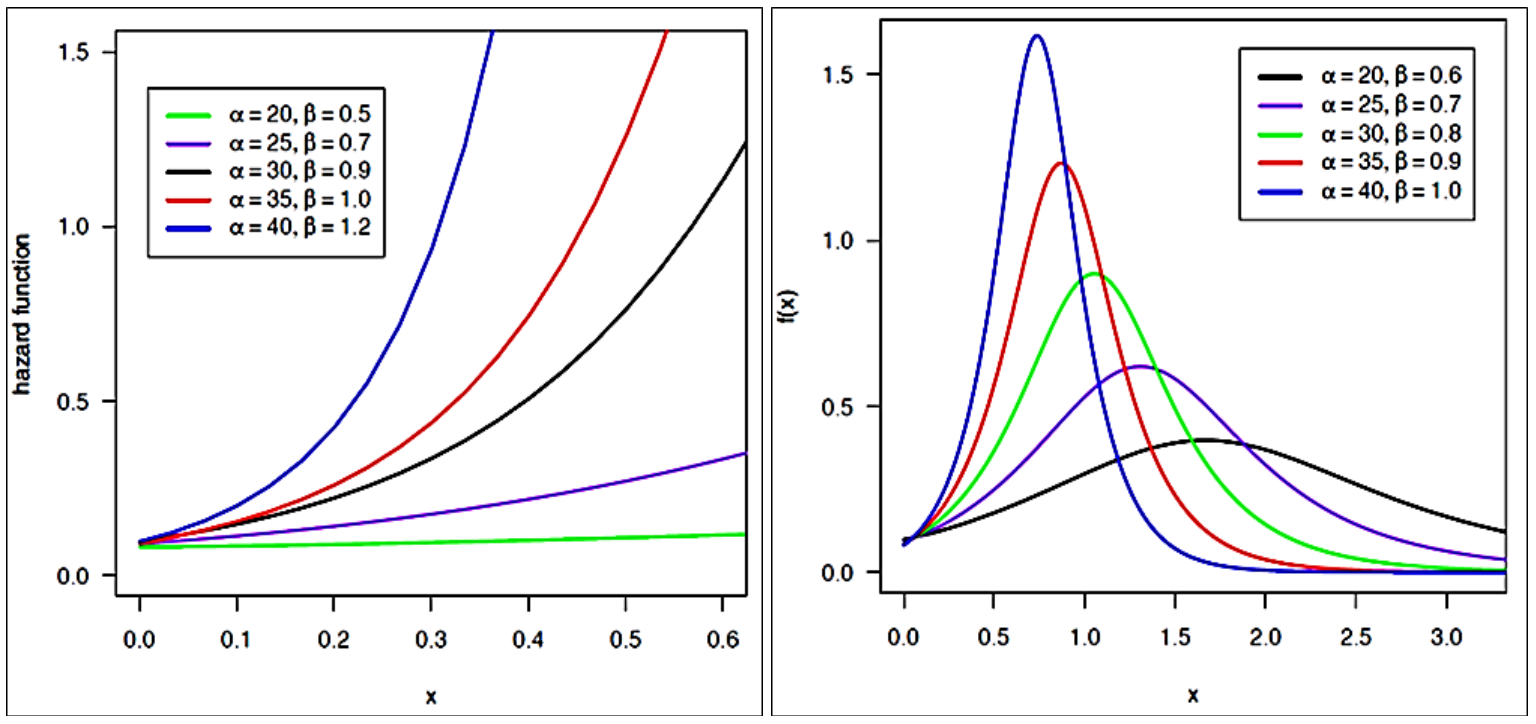

Fig 1: For varying $\alpha$ and $\beta$ values, graphs of hazard function (left panel) and PDF (right panel)

\section{Quantile function}

ATEE distribution's quantile function where p follows uniform distribution $(0,1)$ is

$x_{p}=\frac{1}{\lambda}\left[\{1-\ln (1-z)\}^{1 / \beta}-1\right] ; 0<p<1$

Where $z=1-\frac{1}{\alpha} \arctan \{(1-p) \arctan (\alpha)\}$

\section{Random deviation generation}

$x_{u}=\frac{1}{\lambda}\left[\{1-\ln (1-z)\}^{1 / \beta}-1\right] ; 0<u<1$

Where $z=1-\frac{1}{\alpha} \arctan \{(1-u) \arctan (\alpha)\}$

\section{Skewness and Kurtosis:}

Based on quantiles, Bowley's coefficient of skewness is

$$
\Upsilon_{S k}=\frac{Q(3 / 4)+Q(1 / 4)-2 Q(2 / 4)}{Q(3 / 4)-Q(1 / 4)} \text {, and }
$$

Coefficient of kurtosis based on octiles given by (Moors, 1988) is

$$
M_{\text {Ки }}=\frac{Q(7 / 8)-Q(5 / 8)+Q(3 / 8)-Q(1 / 8)}{Q(3 / 4)-Q(1 / 4)}
$$




\section{Estimation methods}

\subsection{Maximum likelihood estimation method}

For the estimation of the parameter, the maximum likelihood method is the most commonly used method introduced by

(Casella \& Berger, 1990). Let, random sample be denoted by $x_{1}, x_{2}, \ldots, x_{n}$ from $\operatorname{ATEE}(\alpha, \beta, \lambda)$ and the likelihood function, $L(\alpha, \beta, \lambda)$ is given by,

$$
\begin{aligned}
& L\left(\psi ; x_{1}, x_{2} \ldots x_{n}\right)=f\left(x_{1}, x_{2}, \ldots x_{n} / \psi\right)=\prod_{i=1}^{n} f\left(x_{i} / \psi\right) \\
& L(\alpha, \beta, \lambda)=\frac{\alpha \beta \lambda}{\arctan (\alpha)} \prod_{i=1}^{n} \frac{\left(1+\lambda x_{i}\right)^{\beta-1} \exp \left\{1-\left(1+\lambda x_{i}\right)^{\beta}\right\}}{1+\left\{\alpha \exp \left\{1-\left(1+\lambda x_{i}\right)^{\beta}\right\}^{2}\right.} ;(\alpha, \beta, \lambda)>0, x>0
\end{aligned}
$$

Now log-likelihood density is

$$
\begin{aligned}
l=n \ln \alpha+n \ln \beta+n \ln \lambda-n \ln \{\arctan (\alpha)\}-(\beta-1) \sum_{i=1}^{n} \ln \left(1+\lambda x_{i}\right)+n-\sum_{i=1}^{n}\left(1+\lambda x_{i}\right)^{\beta}- \\
\sum_{i=1}^{n} \ln \left\{1+\left[\alpha \exp \left\{1-\left(1+\lambda x_{i}\right)^{\beta}\right\}\right]^{2}\right\}
\end{aligned}
$$

Differentiation of (3.1.1) w.r.t. $\alpha, \beta$ and $\lambda$ we acquire,

$$
\begin{aligned}
& \frac{\partial l}{\partial \alpha}=\frac{n}{\alpha}-\frac{n}{\arctan (\alpha)\left[1+\alpha^{2}\right]}-2 \alpha \sum_{i=1}^{n} \frac{\exp \left\{1-\left(1+\lambda x_{i}\right)^{\beta}\right\}}{\left.1+\left[\alpha \exp \left\{1-\left(1+\lambda x_{i}\right)^{\beta}\right\}\right]^{2}\right]} \\
& \frac{\partial l}{\partial \beta}=\frac{n}{\beta}+\sum_{i=1}^{n} \ln \left(1+\lambda x_{i}\right)-\sum_{i=1}^{n}\left(1+\lambda x_{i}\right)^{\beta} \ln \left(1+\lambda x_{i}\right)+2 \alpha \sum_{i=1}^{n} \frac{\exp 2\left\{1-\left(1+\lambda x_{i}\right)^{\beta}\right\}\left(1+\lambda x_{i}\right)^{\beta} \ln \left(1+\lambda x_{i}\right)}{1+\left[\alpha \exp \left\{1-\left(1+\lambda x_{i}\right)^{\beta}\right\}\right]^{2}} \\
& \frac{\partial l}{\partial \lambda}=\frac{n}{\lambda}-(\beta-1) \sum_{i=1}^{n} \frac{x_{i}}{\left(1+\lambda x_{i}\right)}-\beta \sum_{i=1}^{n} x_{i} \ln \left(1+\lambda x_{i}\right)^{\beta-1}+2 \alpha \beta \sum_{i=1}^{n} \frac{x_{i} \exp 2\left\{1-\left(1+\lambda x_{i}\right)^{\beta}\right\}\left(1+\lambda x_{i}\right)^{\beta-1}}{1+\left[\alpha \exp \left\{1-\left(1+\lambda x_{i}\right)^{\beta}\right\}\right]^{2}}
\end{aligned}
$$

Solving these non-linear functions for $(\alpha, \beta, \lambda)$ by equating to zero we will obtain the ML estimators of the ATEE distribution. The computer softwares like R, Mathmatica, Matlab etc. can be used for maximization of (3.1.1) for obtaining unknown parameter's estimated values. We derive observed information matrix for the parameters for confidence interval estimation of $\alpha, \beta$ and $\lambda$ and hypothesis testing. For $\alpha, \beta$ and $\lambda$, the observed information matrix is as follows

$$
U=\left[\begin{array}{lll}
U_{11} & U_{12} & U_{13} \\
U_{21} & U_{22} & U_{23} \\
U_{31} & U_{32} & U_{33}
\end{array}\right]
$$

Where

$$
\begin{aligned}
& U_{11}=\frac{\partial^{2} l}{\partial \alpha^{2}}, U_{12}=\frac{\partial^{2} l}{\partial \alpha \partial \beta}, U_{13}=\frac{\partial^{2} l}{\partial \alpha \lambda} \\
& U_{21}=\frac{\partial^{2} l}{\partial \beta \partial \alpha}, U_{22}=\frac{\partial^{2} l}{\partial \beta^{2}}, U_{23}=\frac{\partial^{2} l}{\partial \beta \partial \lambda} \\
& U_{31}=\frac{\partial^{2} l}{\partial \lambda \partial \alpha}, U_{32}=\frac{\partial^{2} l}{\partial \beta \partial \lambda}, U_{33}=\frac{\partial^{2} l}{\partial \lambda^{2}}
\end{aligned}
$$


Let $\Omega=(\alpha, \beta, \lambda)$ denote the parameter space and the corresponding MLE of $\Omega$ as $\hat{\Omega}=(\hat{\alpha}, \hat{\beta}, \hat{\lambda})$, then $(\hat{\Omega}-\Omega) \rightarrow N_{3}\left[0,(U(\Omega))^{-1}\right]$ where $U(\Omega)$ is the information matrix of Fisher. Via the algorithm of Newton-Raphson, maximization of likelihood gives the observed information matrix and the var-cov matrix is,

$[U(\Omega)]^{-1}=\left(\begin{array}{ccc}\operatorname{var}(\hat{\alpha}) & \operatorname{cov}(\hat{\alpha}, \hat{\beta}) & \operatorname{cov}(\hat{\alpha}, \hat{\lambda}) \\ \operatorname{cov}(\hat{\alpha}, \hat{\beta}) & \operatorname{var}(\hat{\beta}) & \operatorname{cov}(\hat{\beta}, \hat{\lambda}) \\ \operatorname{cov}(\hat{\alpha}, \hat{\lambda}) & \operatorname{cov}(\hat{\beta}, \hat{\lambda}) & \operatorname{var}(\hat{\lambda})\end{array}\right)$

Thus for $\alpha, \beta$ and $\lambda$, using MLEs' asymptotic normality, approximate $100(1-\alpha) \%$ confidence intervals is given as

$\hat{\alpha} \pm Z_{\alpha / 2} S E(\hat{\alpha}), \hat{\beta} \pm Z_{\alpha / 2} S E(\hat{\beta})$ and $\hat{\lambda} \pm Z_{\alpha / 2} S E(\hat{\lambda})$

Here upper percentile of standard normal variate is denoted by $Z_{\alpha / 2}$.

\subsection{LSE method}

The another estimation method we have used is least-square estimation Swain et al. (1988) to estimate ATEE distribution's $\alpha$, $\beta$ and $\lambda$ which is calculated with minimization of

$$
M(X ; \alpha, \beta, \lambda)=\sum_{i=1}^{n}\left[F\left(X_{i}\right)-\frac{i}{n+1}\right]^{2}
$$

W.R.T. $\alpha, \beta$ and $\lambda$.

From a distribution function $\mathrm{F}\left(\right.$.), suppose $F\left(X_{i}\right)$ represents ordered random variables $\left(\mathrm{X}_{(1)}<\mathrm{X}_{(2)}<\ldots<\mathrm{X}_{(\mathrm{n})}\right)$ 's CDF and random sample is denoted as $\left\{X_{1}, X_{2}, \ldots, X_{n}\right\}$ with "n" size. The LSEs $(\hat{\alpha}, \hat{\beta}$, and $\hat{\lambda})$ is acquired with minimization of

$M(X ; \alpha, \beta, \lambda)=\sum_{i=1}^{n}\left[1-\frac{\arctan \left[\alpha \exp \left\{1-\left(1+\lambda x_{i}\right)^{\beta}\right\}\right]}{\arctan (\alpha)}-\frac{i}{n+1}\right]^{2} ; x \geq 0,(\alpha, \beta, \lambda)>0$.

W.R.T. $\alpha, \beta$ and $\lambda$.

Differentiation of (3.2.2) w.r.t. $\alpha, \beta$ and $\lambda$ we obtain,

$$
\begin{aligned}
& \frac{\partial M}{\partial \alpha}=-2 \sum_{i=1}^{n}\left[1-\frac{\arctan \left[\alpha A\left(x_{i}\right)\right]}{\arctan (\alpha)}-\frac{i}{n+1}\right]\left[\frac{A\left(x_{i}\right)}{\left(\alpha^{2}\left\{A\left(x_{i}\right)\right\}^{2}+1\right) \arctan (\alpha)}-\frac{\arctan \left[\alpha A\left(x_{i}\right)\right]}{\left(\alpha^{2}+1\right) \arctan ^{2}(\alpha)}\right] \\
& \frac{\partial M}{\partial \beta}=\frac{2 \alpha}{\arctan (\alpha)} \sum_{i=1}^{n}\left[1-\frac{\arctan \left[\alpha A\left(x_{i}\right)\right]}{\arctan (\alpha)}-\frac{i}{n+1}\right]\left[\frac{A\left(x_{i}\right)\left(1+\lambda x_{i}\right)^{\beta} \ln \left(1+\lambda x_{i}\right)}{\left(\alpha^{2}\left\{A\left(x_{i}\right)\right\}^{2}+1\right)}\right] \\
& \frac{\partial M}{\partial \lambda}=\frac{2 \alpha \beta}{\arctan (\alpha)} \sum_{i=1}^{n}\left[1-\frac{\arctan \left[\alpha A\left(x_{i}\right)\right]}{\arctan (\alpha)}-\frac{i}{n+1}\right]\left[\frac{x_{i}\left(1+\lambda x_{i}\right)^{\beta-1} A\left(x_{i}\right)}{\left(\alpha^{2}\left\{w\left(x_{i}\right)\right\}^{2}+1\right)}\right]
\end{aligned}
$$

Where $A\left(x_{i}\right)=\exp \left\{1-\left(1+\lambda x_{i}\right)^{\beta}\right\}$

Likewise weighted LSEs is given with minimization of

$$
M(X ; \alpha, \beta, \lambda)=\sum_{i=1}^{n} w_{i}\left[F\left(X_{(i)}\right)-\frac{i}{n+1}\right]^{2}
$$


W.R.T. $\alpha, \beta$ and $\lambda$. The weights $w_{i}$ are $w_{i}=\frac{1}{\operatorname{Var}\left(X_{(i)}\right)}=\frac{(n+1)^{2}(n+2)}{i(n-i+1)}$

Minimization of the following equation

$$
M(X ; \alpha, \beta, \lambda)=\sum_{i=1}^{n} \frac{(n+1)^{2}(n+2)}{i(n-i+1)}\left[1-\frac{\arctan \left[\alpha \exp \left\{1-\left(1+\lambda x_{i}\right)^{\beta}\right\}\right]}{\arctan (\alpha)}-\frac{i}{n+1}\right]^{2}
$$

W.R.T. $\alpha, \beta$ and $\lambda$ gives the unknown parameter's weighted least square estimators

\subsection{CVME method}

The CVM estimators of unknown parameter can be attained with minimization of

$$
\begin{aligned}
& D(X ; \alpha, \beta, \lambda)=\frac{1}{12 n}+\sum_{i=1}^{n}\left[F\left(x_{i: n} \mid \alpha, \beta, \lambda\right)-\frac{2 i-1}{2 n}\right]^{2} \\
& =\frac{1}{12 n}+\sum_{i=1}^{n}\left[1-\frac{\arctan \left[\alpha \exp \left\{1-\left(1+\lambda x_{i}\right)^{\beta}\right\}\right]}{\arctan (\alpha)}-\frac{2 i-1}{2 n}\right]^{2}
\end{aligned}
$$

Differentiation of (3.4.1) w.r.t. $\alpha, \beta$ and $\lambda$ we obtain,

$$
\begin{aligned}
& \frac{\partial D}{\partial \alpha}=-2 \sum_{i=1}^{n}\left[1-\frac{\arctan \left[\alpha A\left(x_{i}\right)\right]}{\arctan (\alpha)}-\frac{2 i-1}{2 n}\right]\left[\frac{A\left(x_{i}\right)}{\left(\alpha^{2}\left\{A\left(x_{i}\right)\right\}^{2}+1\right) \arctan (\alpha)}-\frac{\arctan \left[\alpha A\left(x_{i}\right)\right]}{\left(\alpha^{2}+1\right) \arctan ^{2}(\alpha)}\right] \\
& \frac{\partial D}{\partial \beta}=\frac{2 \alpha}{\arctan (\alpha)} \sum_{i=1}^{n}\left[1-\frac{\arctan \left[\alpha A\left(x_{i}\right)\right]}{\arctan (\alpha)}-\frac{2 i-1}{2 n}\right]\left[\frac{A\left(x_{i}\right)\left(1+\lambda x_{i}\right)^{\beta} \ln \left(1+\lambda x_{i}\right)}{\left(\alpha^{2}\left\{A\left(x_{i}\right)\right\}^{2}+1\right)}\right] \\
& \frac{\partial D}{\partial \lambda}=\frac{2 \alpha \beta}{\arctan (\alpha)} \sum_{i=1}^{n}\left[1-\frac{\arctan \left[\alpha A\left(x_{i}\right)\right]}{\arctan (\alpha)}-\frac{2 i-1}{2 n}\right]\left[\frac{x_{i}\left(1+\lambda x_{i}\right)^{\beta-1} A\left(x_{i}\right)}{\left(\alpha^{2}\left\{w\left(x_{i}\right)\right\}^{2}+1\right)}\right]
\end{aligned}
$$

Where $A\left(x_{i}\right)=\exp \left\{1-\left(1+\lambda x_{i}\right)^{\beta}\right\}$

After solving non-linear equations $\frac{\partial D}{\partial \alpha}=0, \frac{\partial D}{\partial \beta}=0$ and $\frac{\partial D}{\partial \lambda}=0$ CVM estimators can be obtained

\section{Applications to real dataset}

In this section, we demonstrate the applicability of arctan exponential extension distribution with the help of a real dataset used (Oguntunde et al., 2015) ${ }^{[18]}$. The dataset is on the breaking stress of carbon fibres of $50 \mathrm{~mm}$ length (GPa). The dataset is as follows:

0.39, $0.85,1.08,1.25,1.47,1.57,1.61,1.61,1.69,1.80,1.84,1.87,1.89,2.03,2.03,2.05,2.12,2.35,2.41,2.43,2.48,2.50$, 2.53, 2.55, 2.55, 2.56, 2.59, 2.67, 2.73, 2.74, 2.79, 2.81, 2.82, 2.85, 2.87, 2.88, 2.93, 2.95, 2.96, 2.97, 3.09, 3.11, 3.11, 3.15, 3.15, 3.19, 3.22, 3.22, 3.27, 3.28, 3.31, 3.31, 3.33, 3.39, 3.39, 3.56, 3.60, 3.65, 3.68, 3.70, 3.75, 4.20, 4.38, 4.42, 4.70, 4.90 By utilizing R software (R Core Team, 2020) of the optim() function, we have calculated the MLEs of ATEE distribution by maximizing the likelihood function (3.1.1) (Mailund, 2017) where Log-Likelihood's values was obtained as $l=-84.6891$. For $\alpha, \beta$, and $\lambda$ MLE's with their standard errors (SE) has been illustrated in Table 1.

Table 1: MLE and SE for $\alpha, \beta$ and $\lambda$

\begin{tabular}{|c|c|c|}
\hline Parameter & MLE & SE \\
\hline alpha & 17.6872 & 4.9779 \\
\hline beta & 3.3043 & 2.2682 \\
\hline lambda & 0.1827 & 0.1627 \\
\hline
\end{tabular}


Log-likelihood function's plot for $\alpha, \beta$ and $\lambda$ has been illustrated in Figure 4 (Kumar \& Ligges, 2011) ${ }^{[19]}$ and found that the ML estimates can be calculated uniquely.
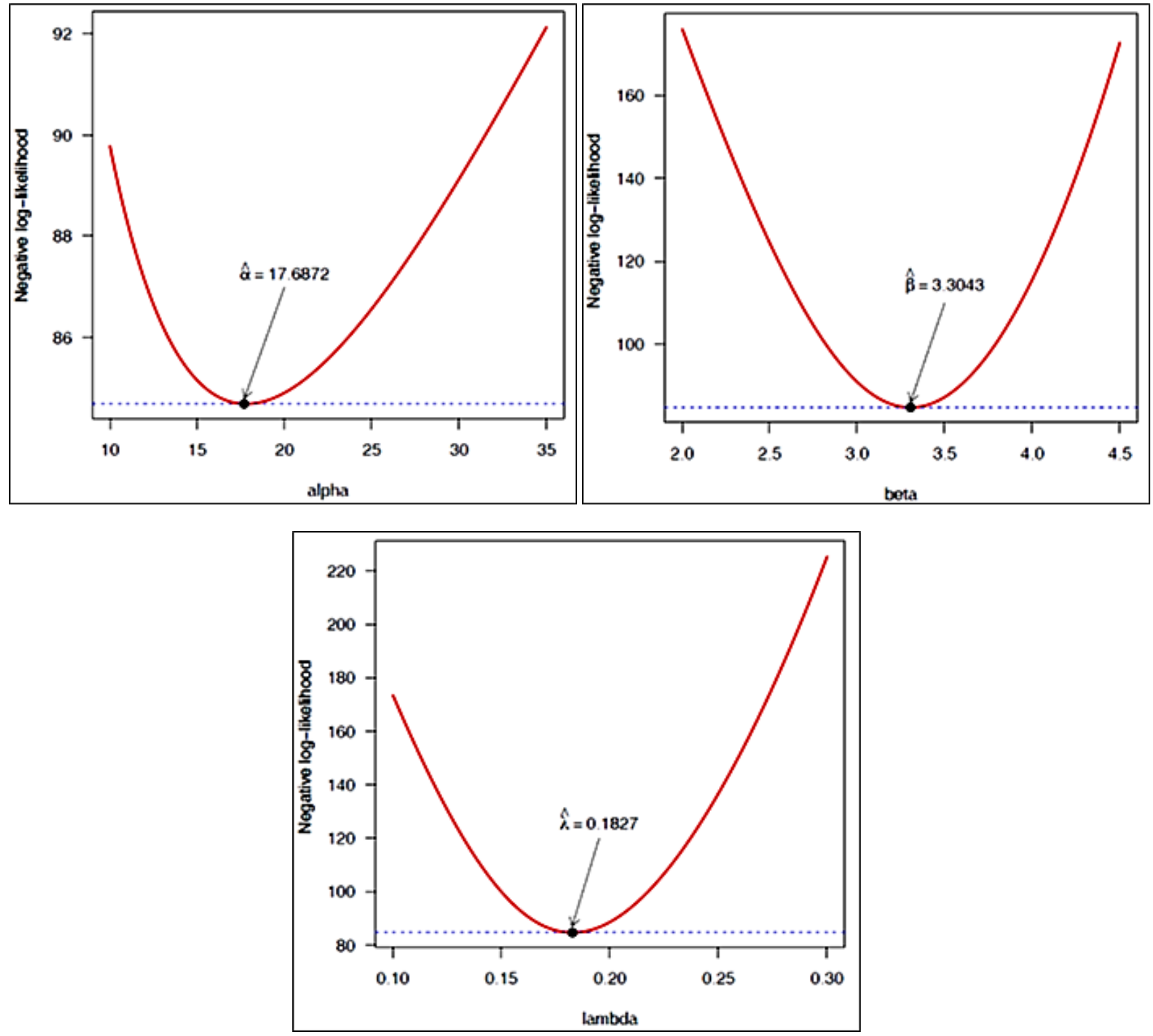

Fig 2: Profile log-likelihood function's graphs of $\alpha, \beta$, and $\lambda$

In Figure 3 plots of Q-Q and P-P of ATEE distribution has been illustrated.
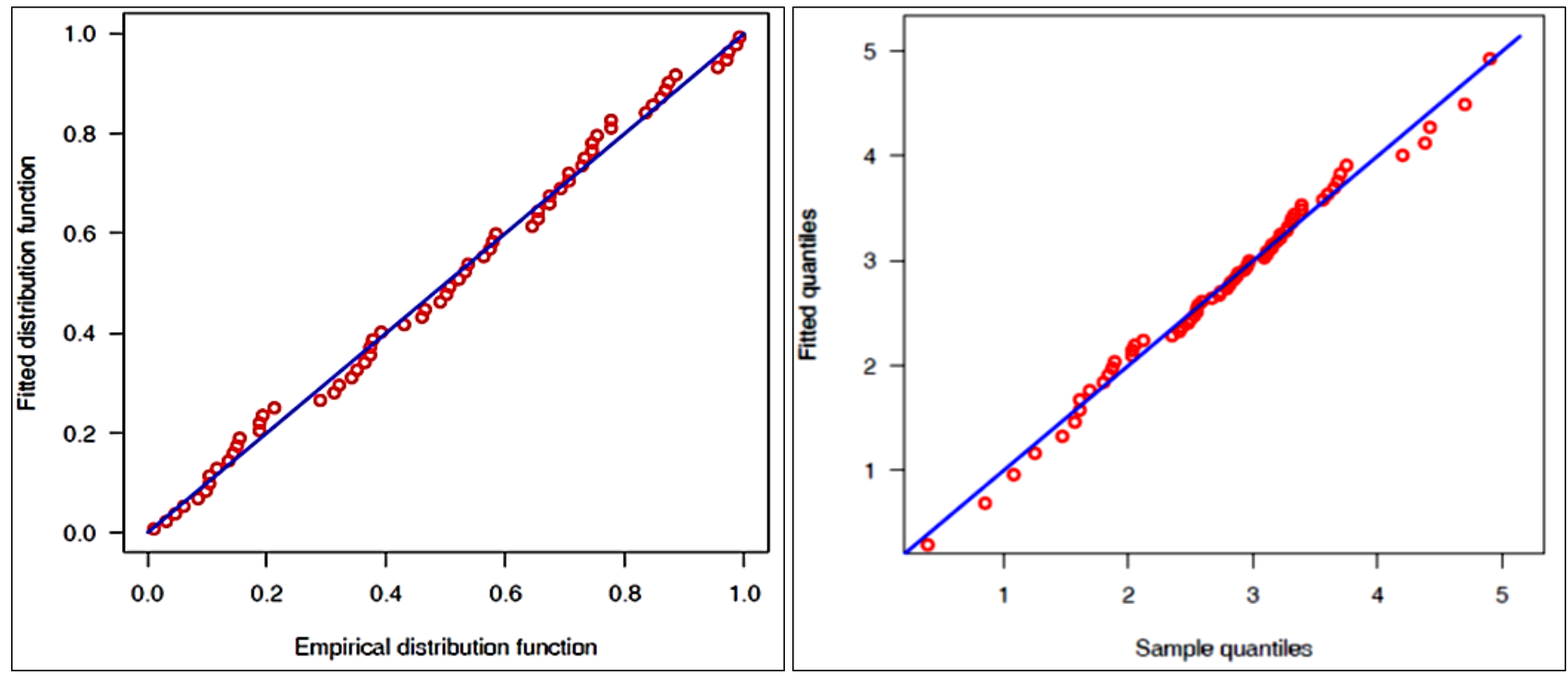

Fig 3: The ATEE distribution's plots of P-P (left panel) and Q-Q (right panel) $\sim 438 \sim$ 
In Table 2 the parameters' estimated values of ATEE distribution using LSE, MLE and CVE method and their negative loglikelihood, AIC and KS criterion has been given.

Table 2: Estimated parameters, log-likelihood, AIC, BIC and KS (p-value)

\begin{tabular}{|c|c|c|c|c|c|c|c|}
\hline Method of Estimation & $\hat{\alpha}$ & $\hat{\beta}$ & $\hat{\lambda}$ & -LL & AIC & BIC & KS(p-value) \\
\hline MLE & 17.6872 & 3.3043 & 0.1827 & 84.6891 & 175.3781 & 181.9471 & $0.0568(0.9836)$ \\
\hline LSE & 13.7865 & 6.8864 & 0.0742 & 84.8314 & 175.6628 & 182.2318 & $0.0549(0.9886)$ \\
\hline CVE & 14.7207 & 6.9800 & 0.0741 & 84.9010 & 175.8020 & 182.3710 & $0.0543(0.9900)$ \\
\hline
\end{tabular}

In Figure 3 we have plotted the histogram and the density function of fitted distributions and Q-Q plot of estimation methods MLE, LSE and CVM for ATEE distribution.
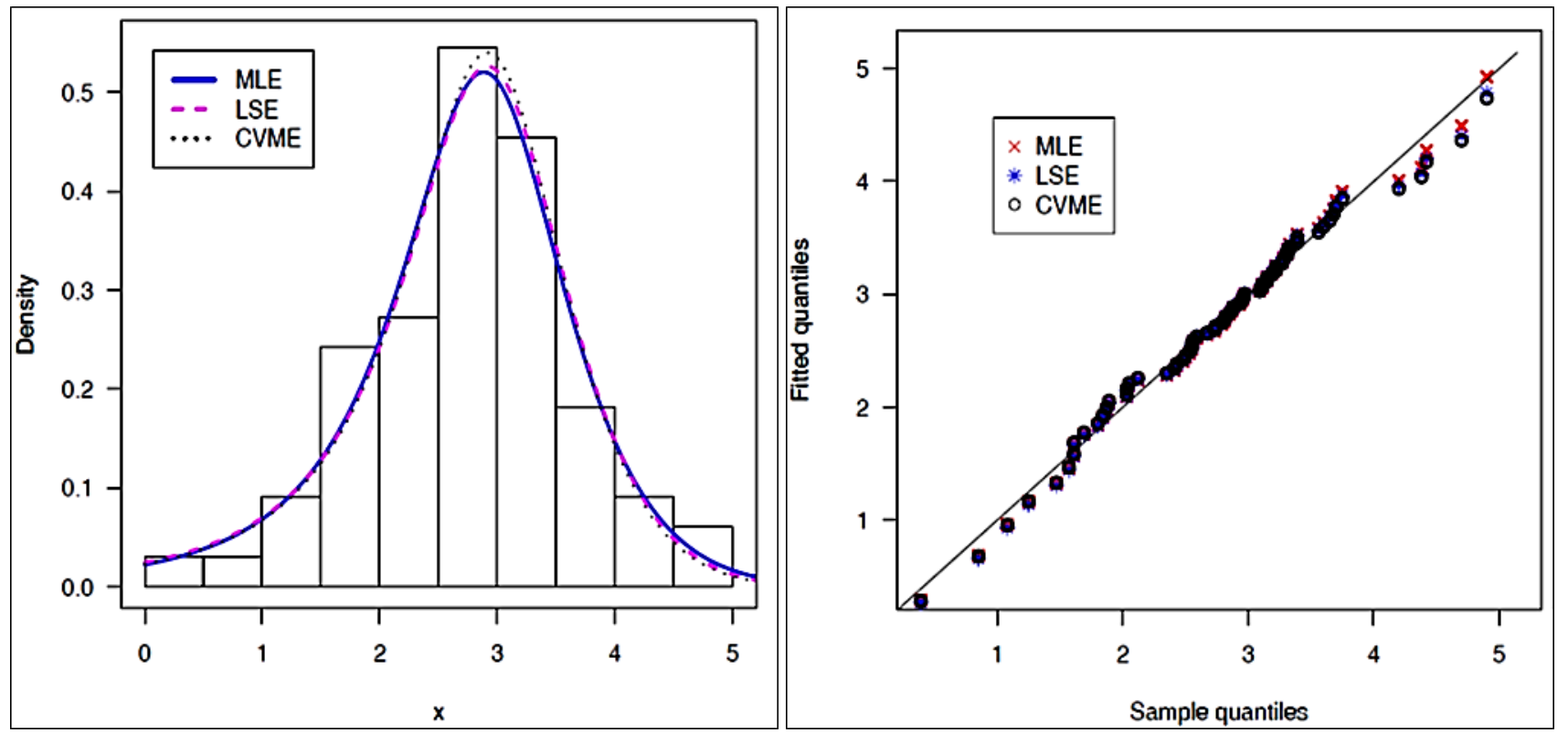

Fig 3: The Histogram and the density function of distributions fitted of MLE, LSE and CVM (left panel) and fitted quantiles and sample quantiles (right panel) of ATEE distribution

For comparison purpose to evaluate goodness-of-fit of ATEE distribution, following models are taken.

\section{A. Generalized gompertz (GG) distribution}

The PDF of GG distribution (El-Gohary et al., 2013) ${ }^{[11]}$ with parameters $\alpha, \lambda$ and $\theta$ is

$$
f_{G G Z}(x)=\theta \lambda e^{\alpha x} e^{-\frac{\lambda}{\alpha}\left(e^{\alpha x}-1\right)}\left[1-\exp \left(-\frac{\lambda}{\alpha}\left(e^{\alpha x}-1\right)\right)\right]^{\theta-1} ; \lambda, \theta>0, \alpha \geq 0, x \geq 0
$$

\section{B. Exponentiated exponential poisson (EEP)}

The probability density function of EEP (Ristić \& Nadarajah, 2014) ${ }^{[31]}$ can be expressed as

$$
f(x)=\frac{\alpha \beta \lambda}{\left(1-e^{-\lambda}\right)} e^{-\beta x}\left(1-e^{-\beta x}\right)^{\alpha-1} \exp \left\{-\lambda\left(1-e^{-\beta x}\right)^{\alpha}\right\} ; x>0, \alpha>0, \lambda>0
$$

\section{Generalized Exponential Extension (GEE) distribution:}

GEE's PDF given by (Lemonte, 2013) ${ }^{[21]}$ with parameters $\alpha, \beta$ and $\lambda$ is

$$
\begin{aligned}
& f_{G E E}(x ; \alpha, \beta, \lambda)=\alpha \beta \lambda(1+\lambda x)^{\alpha-1} \exp \left\{1-(1+\lambda x)^{\alpha}\right\} \\
& {\left[1-\exp \left\{1-(1+\lambda x)^{\alpha}\right\}\right]^{\beta-1} ; x \geq 0 . }
\end{aligned}
$$




\section{Exponential power (EP) distribution:}

EP distribution's PDF (Smith \& Bain, 1975) is

$$
f_{E P}(x)=\alpha \lambda^{\alpha} x^{\alpha-1} e^{(\lambda x)^{\alpha}} \exp \left\{1-e^{(\lambda x)^{\alpha}}\right\} ;(\alpha, \lambda)>0, \quad x \geq 0
$$

Where $\alpha$ and $\lambda$ are the shape and scale parameters.

\section{E. Generalized Rayleigh (GR) distribution}

GR distribution's PDF (Kundu \& Raqab, 2005) ${ }^{[20]}$ is

$$
f_{G R}(\mathrm{x} ; \alpha, \lambda)=2 \alpha \lambda^{2} \mathrm{xe}^{-(\lambda x)^{2}}\left\{1-\mathrm{e}^{-(\lambda x)^{2}}\right\}^{\alpha-1} ;(\alpha, \lambda)>0, x>0
$$

Here $\alpha$ and $\lambda$ are the shape and scale parameters respectively.

We have illustrated the Bayesian information criterion (BIC), Akaike information criterion (AIC), Hannan-Quinn information criterion (HQIC) and Corrected Akaike information criterion (CAIC) for the evaluation of the applicability of the ATEE distribution in Table 4.

Table 3: Log-likelihood (LL), AIC, BIC, CAIC and HQIC

\begin{tabular}{|c|c|c|c|c|c|}
\hline Distribution & LL & AIC & BIC & CAIC & HQIC \\
\hline ATEE & -84.6891 & 175.3781 & 181.9471 & 175.7652 & 177.9738 \\
\hline GGZ & -85.6858 & 177.3716 & 183.9406 & 177.7587 & 179.9673 \\
\hline EEP & -86.7885 & 179.5770 & 186.1460 & 179.9641 & 182.1727 \\
\hline GEE & -87.2705 & 180.5409 & 187.1099 & 180.9280 & 183.1366 \\
\hline EP & -87.3974 & 178.7949 & 183.1742 & 178.9795 & 180.5254 \\
\hline GR & -88.6368 & 181.2735 & 185.6528 & 181.4640 & 183.0040 \\
\hline
\end{tabular}

We have presented the plot of goodness-of-fit of PSG distribution and some selected distributions are in Figure 4.
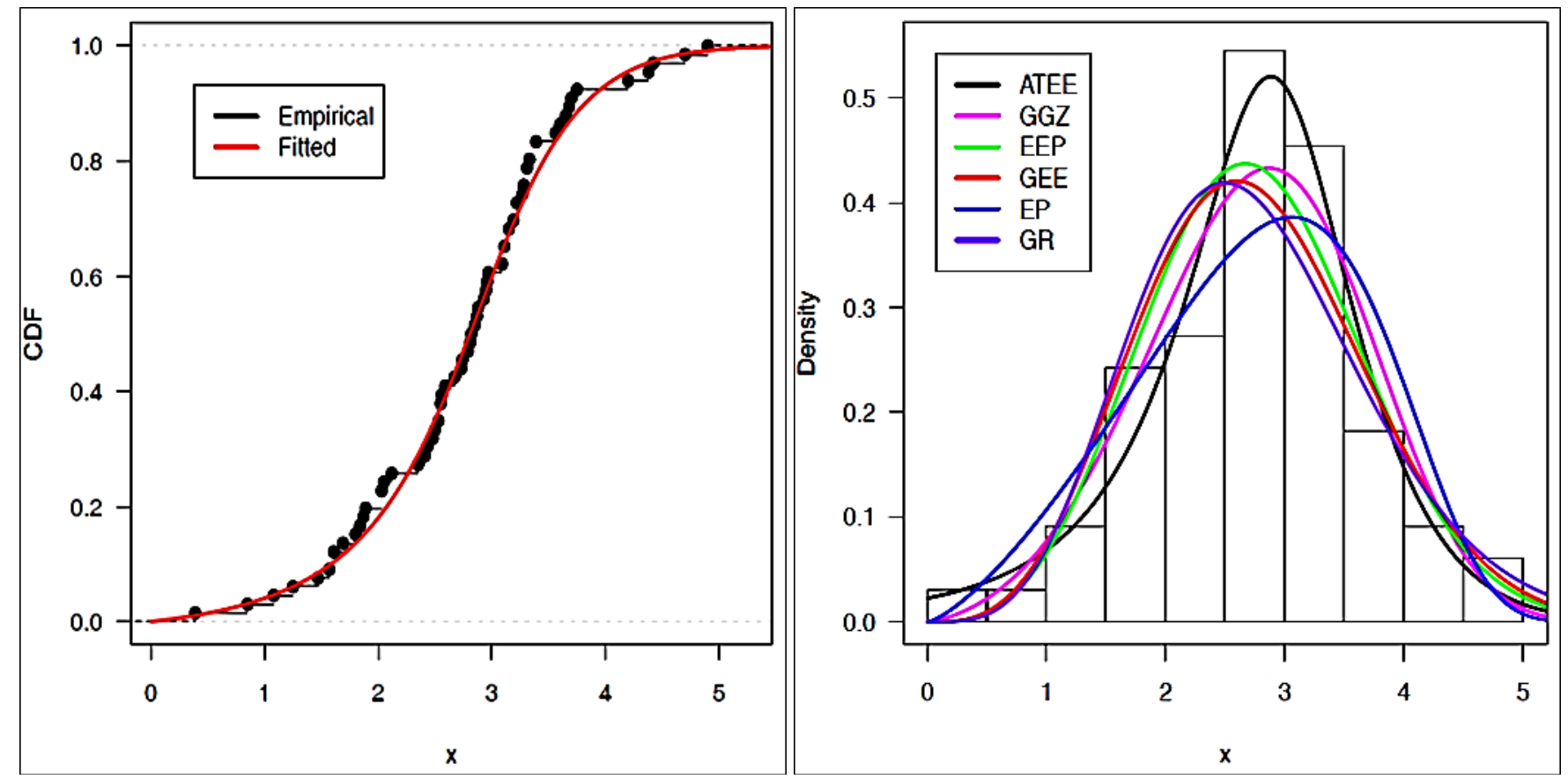

Fig 4: Empirical distribution function with estimated distribution function (Left) and The Histogram and the density function of fitted distributions (Right)

To compare the ATEE distribution's goodness of fit among different models, different values of goodness of fit statistics are given in Table 5 where the test statistics for model purposed was observed to have low value also the p-value was higher. Thus conclusion that ATEE distribution shows better fit with more reliability and consistency in results among others taken for comparison. 
Table 4: The goodness-of-fit statistics and their p-value

\begin{tabular}{|c|c|c|c|}
\hline Distribution & $\mathbf{W}(\mathbf{p}$-value) & KS(p-value) & $\mathbf{A}^{\mathbf{2}}$ (p-value) \\
\hline ATEE & $0.0305(0.9755)$ & $0.0568(0.9836)$ & $0.2232(0.9827)$ \\
\hline GGZ & $0.0715(0.7443)$ & $0.0833(0.7498)$ & $0.4457(0.8020)$ \\
\hline EEP & $0.1098(0.5403)$ & $0.0931(0.6160)$ & $0.5980(0.6492)$ \\
\hline GEE & $0.1541(0.3780)$ & $0.1099(0.4021)$ & $0.7853(0.4913)$ \\
\hline EP & $0.1434(0.4111)$ & $0.1126(0.3729)$ & $0.8796(0.4266)$ \\
\hline GR & $0.1948(0.2781)$ & $0.1205(0.2931)$ & $1.0049(0.3544)$ \\
\hline
\end{tabular}

\section{Conclusions}

In this article, arctan exponential extension distribution, a univariate continuous distribution, is presented. A comprehensive study of some distributional characteristics of the new distribution is presented build a clearer picture for the distribution purposed. Parameter estimation is carried out with MLE along with CVME and LSE. The proposed distribution's applicability and suitability has been evaluated by considering a real set of data and the results exposed that the proposed distribution is much flexible in comparison to some other fitted distributions which leads us to hope this model, in survival analysis, may prove to be an alternative.

\section{References}

1. Abdulkabir M, Ipinyomi RA. Type ii half logistic exponentiated exponential distribution: properties and applications. Pakistan Journal of Statistics, 2020, 36(1).

2. Abouammoh AM, Alshingiti AM. Reliability estimation of generalized inverted exponential distribution. Journal of Statistical Computation and Simulation 2009;79(11):1301-1315.

3. Almarashi AM, Elgarhy M, Elsehetry MM, Kibria BG, Algarni A. A new extension of exponential distribution with statistical properties and applications. Journal of Nonlinear Sciences and Applications 2019;12:135-145.

4. Barreto-Souza W, Santos AHS, Cordeiro GM. The beta generalized exponential distribution. Journal of Statistical Computation and Simulation 2010;80(2):159-172.

5. Casella G, Berger RL. Statistical Inference. Brooks/ ole Publishing Company, California 1990.

6. Chaudhary AK, Kumar V. Half logistic exponential extension distribution with Properties and Applications. International Journal of Recent Technology and Engineering (IJRTE) 2020:8(3):506-512.

7. Chaudhary AK Kumar. The Logistic Exponential Extension Distribution with Properties and Applications. International Journal of Latest Trends In Engineering and Technology (IJLTET) 2020;18(2):20-30.

8. Chaudhary AK, Kumar V. New Exponentiated Exponential Extension Distribution with Properties and Applications. International Journal of Innovative Research in Science, Engineering and Technology (IJIRSET) 2020;9(12):11370-11379.

9. Chaudhary AK, Sapkota LP, Kuma V. Truncated Cauchy power-exponential distribution: Theory and Applications. IOSR Journal of Mathematics (IOSR-JM), Ser. I 2020;16(6):44-52.

10. Cordeiro GM, De Castro M. A new family of generalized distributions. Journal of Statistical Computation and Simulation 2011;81:883-898.

11. El-Gohary A, Alshamrani A, Al-Otaibi AN. The generalized Gompertz distribution. Applied Mathematical Modelling 2013;37(1-2):13-24.

12. Gupta RD, Kundu D. Generalized exponential distribution: Existing results and some recent developments. Journal of Statistical Planning and Inference 2007;137(11):3537-3547.

13. Gómez-Déniz E, Calderín-Ojeda E. Modelling insurance data with the Pareto Arc Tan distribution. ASTIN Bulletin: The Journal of the IAA 2015;45(3):639-660.

14. Gomez YM, Bolfarine H, Gomez HW. A new extension of the exponential distribution. Revista Colombiana de Estadistica 2014;37(1):25-34.

15. Hassan AS, Mohamd RE, Elgarhy M, Fayomi A. Alpha power transformed extended exponential distribution: properties and applications. Journal of Nonlinear Sciences and Applications 2018;12(4):62-67.

16. Joshi RK, Kumar V. Lindley exponential power distribution with Properties and Applications. International Journal for Research in Applied Science \& Engineering Technology (IJRASET) 2020;8(10):22-30.

17. Joshi RK, Kumar V. Half Logistic NHE. Properties and Application. International Journal for Research in Applied Science \& Engineering Technology (IJRASET) 2020;8(9):742-753.

18. Kumar V. Bayesian analysis of exponential extension model. J Nat. Acad. Math 2010;24:109-128.

19. Kumar V, Ligges U Reliar. A package for some probability distributions, http://cran.rproject.org/web/packages/reliaR/index.html. 2011.

20. Kundu D, Raqab MZ. Generalized Rayleigh Distribution: Different Methods of Estimation, Computational Statistics and Data Analysis 2005;49:187-200.

21. Lemonte AJ. A new exponential-type distribution with constant, decreasing, increasing, upside-down bathtub and bathtubshaped failure rate function. Computational Statistics \& Data Analysis 2013;62:149-170.

22. Mahdavi A, Kundu D. A new method for generating distributions with an application to exponential distribution. Communications in Statistics-Theory and Methods 2017;46(13):6543-6557.

23. Mailund T. Functional Programming in R: Advanced Statistical Programming for Data Science, Analysis and Finance. Apress, Aarhus N, Denmark 2017. ISBN-13 (pbk): 978-1-4842-2745-9 ISBN-13 (electronic): 978-1-4842-2746-6 DOI 10.1007/978-1-4842-2746-6

24. Merovci F. The transmuted Rayleigh distribution. Australian J of Stat 2013;22(1):21-30. 
25. Moors J. A quantile alternative for kurtosis. The Statistician 1988;37:25-32.

26. Nadarajah S, Haghighi F. An extension of the exponential distribution. Statistics 2011;45(6):543-558.

27. Nadarajah S, Kotz S. The beta exponential distribution. Reliability Engineering and System Safety 2006;91(6):689-697.

28. Oguntunde PE, Balogun OS, Okagbue HI, Bishop SA. The Weibull-exponential distribution: Its properties and applications. Journal of Applied Sciences 2015;15(11):1305-1311.

29. Core Team R. R: A language and environment for statistical computing. R Foundation for Statistical Computing, Vienna, Austria 2020. URL https://www.R-project.org/.

30. Ristic MM, Balakrishnan N. The gamma-exponentiated exponential distribution. Journal of Statistical Computation and Simulation 2012;82(8):1191-1206.

31. Ristić MM, Nadarajah S. A new lifetime distribution. Journal of Statistical Computation and Simulation 2014;84(1):135150.

32. Smith RM, Bain LJ. An exponential power life-test distribution, Communications in Statistics 1975;4:469-481.

33. Swain JJ, Venkatraman S, Wilson JR. Least-squares estimation of distribution functions in johnson's translation system. Journal of Statistical Computation and Simulation 1988;29(4):271-297. 